\title{
An automatic jet fire extinguishing device based on video
}

\author{
Xuejun Chen \\ Department of Mechanical \& Electrical Engineering \\ Putian University \\ Putian, China \\ cxjnet@126.com
}

\author{
Xiaomei Li \\ Department of Mechanical \& Electrical Engineering \\ Putian University \\ Putian, China \\ wxdy5258@sina.com
}

\begin{abstract}
With the increasing large space buildings, the suitability of sprinkler has been challenged using in such large space situations. The automatic jet fire extinguishing devices based on infrared were designed and used to extinguish fires. The accuracy of the devices will significantly reduce in the windy or air flow occasions. An automatic jet fire extinguishing device based on video was developed in the paper. DSP was used for central processing unit of the proposed fire extinguishing device. A key problem of videobased fire extinguishing device is to accurately recognize a fire and estimate the fire centroid. Then a fire detecting and locating method was proposed for a video-based device which was developed our latest work. Moreover, experiments were conducted in a large test hall to verify the video-based automatic jet fire extinguishing device. The results show that this method can locate and extinguish the test fire well.
\end{abstract}

Keywords-fire extinguishing; fire; video; image processing; locating

\section{INTRODUCTION}

The fire has become one of the most horrible killers in modem society. Many wealth and lives are fallen in danger. Many countries have legislated for mandatory installation of smoke detectors in buildings. Consequently, a variety of technologies were developed to prevent and control fires [1-2]. Theses thermal and smoke detectors are widely used nowadays, but they typically take charge of a limited area in space. In large rooms and high buildings, it may take a long time for smoke particles and heat to reach a detector, and even it would not reach the detector.

Therefore, fire detector based on video is studied and developing at present [3-4]. For it can greatly serve the fire detection requirement in large rooms and high buildings, and even outdoor environment. With the fire can be detected and a warning, another important problem need to face. That is precisely how to put out a fire.

Many extinguishing systems were designed and used to fight fires, such as water-based fire extinguishing system and gas extinguisher extinguishing system. From an economic perspective, water has been the most available and the most frequently used extinguishing material. However, that damage resulting from inefficient application of water usually exceeds that done by fire to the burned down property and other valuables [5]. Many researchers and engineers have been trying to find better ways of delivering water to the scene of an accident and fighting fire by the water in the most effective way. Although various up-to-date pumps, nozzles and sprayers are used to extinguish fires, water-based fire extinguishing technologies have not reached the top level of performance.

In recent years, some automatic jet fire extinguishing devices based on infrared were designed and used to extinguish fires [6]. However, in the windy or air flow occasions, the fire suppression accuracy of the devices will significantly reduce. With the development of computer technology, robotic fire monitor was studied which can be automatically controlled by computer and can detect and suppressing fire. Chen et al. [7] has developed an automatic fire searching and suppression system with remote-controlled fire monitors. The fire searching method is realized based on computer vision theory via one CCD camera fixed at the end of a fire monitor chamber. Due to connection interfaces between the detector controller, computer and fire monitor, the total response times of the water cannon, fire scanning time, accuracy of fire targeting are need to improve [8].

In order to improve the fire extinguishing efficiency to adapt to fire fighting requirements, an automatic jet fire extinguishing device based on video was developed in the paper. The proposed fire extinguishing device technology is based on DSP. It reduces connection interfaces between the detector controller and fire monitor. A new fire detecting and location method used for the proposed fire extinguishing device is described. With fire confirmation and tracing process, the proposed fire extinguishing device can easily locate and suppress the fire.

\section{VIDEO-BASED AUTOMATIC JET FIRE- EXTINGUISHING DEVICE}

The automatic jet fire extinguishing device is made up of video acquisition module, sensor module, DSP processor module, communication module and output module. The diagram of the automatic jet fire extinguishing device is shown in Fig .1 (a). There is a camera, which is equipped with a set of wavelength infrared filter, and the output is an infrared video signal. The video acquisition module will capture the infrared scene real time and transmitted them to DSP processing center through video encoder. The DSP processing center will process the received videos, including denoise, fire detecting and locating, and output.

The sensor module comprises some limit switches and two encoder. When the horizontal and vertical rotary 
motors were controlled by relay module, the state of the jet fire extinguishing device will change. Then the current state will be input to DSP in time by the sensor module. The fire alarm and controlling signals would be transmitted to the manual and center controller through the RS485 communication module. The manual controller is used for manual operations at emergency situation. Users in fire control center can send the controller signals to the device with center controller. Once the fire detector is in alarm state, the device would be activated. It would detect and locate the fire, thus open valve to suppress the fire. The made result of the device is shown in Fig .1 (b).

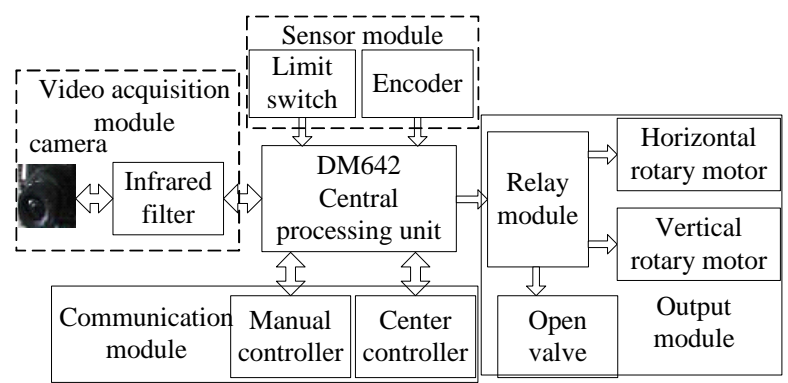

(a) Diagram for proposed device

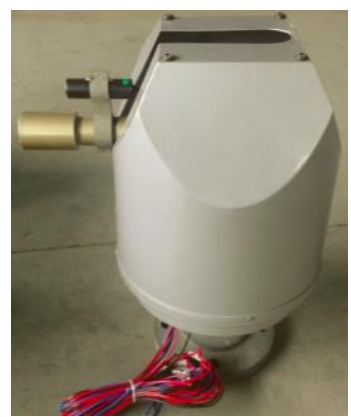

(b) The made result of the device

Figure 1. The automatic jet fire extinguishing device.

\section{FIRE DETECTING AND LOCATING METHOD}

It is crucial for the performance of video-based automatic jet fire extinguishing device to locate a fire center. The aim is to find the coordinates of fire centroid. Then fire position can be obtained by analyzing the coordinates of fire centroid. The proposed fire localization algorithm includes four steps. They include binarization processing, morphology processing, edge detecting, and calculating coordinates of fire centroid. The algorithm flow chart of fire centroid localization and processing is shown in Fig .2.

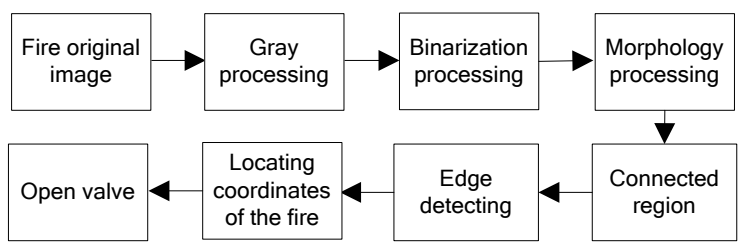

Figure 2. Algorithm flow chart of fire center location.

\section{A. Morphology processing}

In order to detect and segment fire from the original image, the original image should be converted to grayscale images, and then to the binarization images. A frame image of fire videos gained by video acquisition module was shown in Fig .3 (a), and Fig .3 (b) was its grayscale image. The binarization image was shown in Fig .3 (c). Then the binarization image was processed by median filter using different window. The processed results were shown in Fig $.3(\mathrm{~d})$.

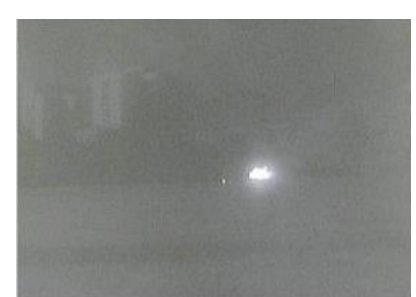

(a) Original fire image

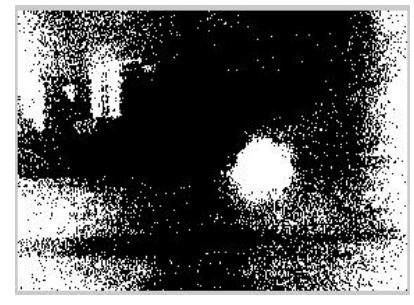

(c) binarizaton image

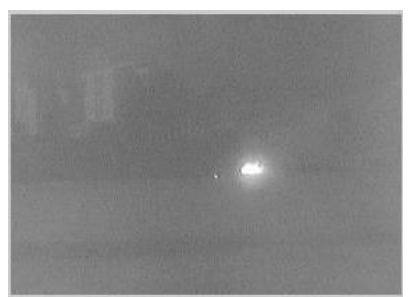

(b) grayscale image

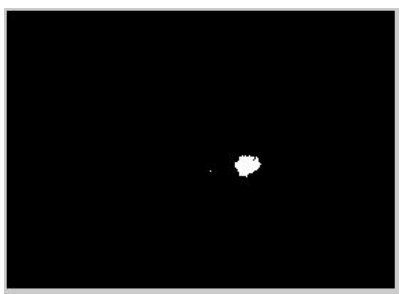

(d) denoising image
Figure 3. Image preprocessing for a frame image of fire videos.

After the original image of a fire has been processed to binarization image, mathematical morphology was used to adaptively process the structuring elements for fire extraction in binarizaton image. Among the operation of mathematical morphology, erosion and dilation is the base of other complicated operations' implementation. Erosion is a transformation of shrinking, which decreases the greyscale value of the image, while dilation is a transformation of expanding, which increases the grey-scale value of the image.

In order to obtain more obvious fire area from binarization image, the closing and opening operations were used to denoise the binarization image and detect edge of fire area. Let $\mathrm{F}(\mathrm{x}, \mathrm{y})$ denote a binarization image, and $\mathrm{B}$ denote structuring element. Opening and closing of $\mathrm{F}(\mathrm{x}, \mathrm{y})$ by $\mathrm{B}$ are denoted respectively by equation (1) and (2) [9-10].

$$
\begin{aligned}
& \mathrm{F} 。 \mathrm{~B}=(\mathrm{F} \Theta \mathrm{B}) \oplus \mathrm{B} \\
& \mathrm{F} \cdot \mathrm{B}=(\mathrm{F} \oplus \mathrm{B}) \Theta \mathrm{B}
\end{aligned}
$$

Opening is erosion followed by dilation and closing is dilation followed by erosion. Opening can smoothe the contour of the binarizaton image and break narrow gaps. Compared by opening, closing tends to fuse narrow breaks, eliminates small holes, and fills gaps in the contours.

When the binarization image was processed by closing and opening at first time, the results were shown in Fig .4 (a) and (b) respectively. In order to obtain better fire area, the image was processed by closing and opening at second time, the results were shown in Fig .4 (c) and (d) respectively. If the last results show that there are several connected regions, and their sizes are different usually. In order to gain fire region, median filter and connected region processing were used for Fig .4 (d). Only was one biggest connected region obtained, which is located fire. Then next step is to get coordinates of fire center. 


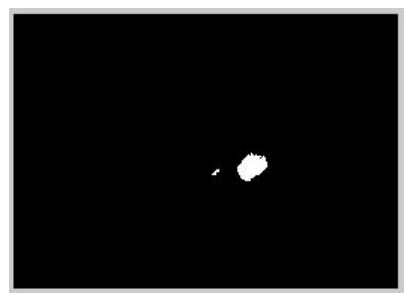

(a) first time dilation

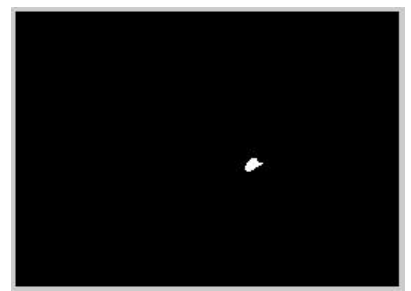

(c) second time erosion

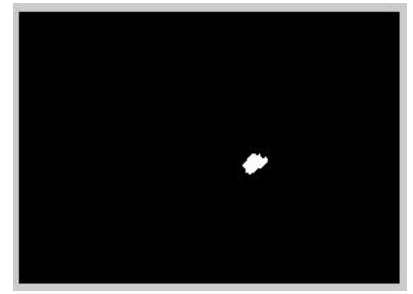

(b) first time erosion

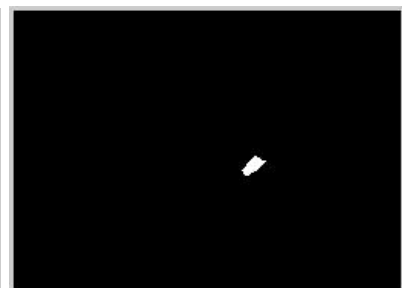

(d) second time dilation
Figure 4. The results of morphology preprocessing.

\section{B. Edge detecting and centroid locating}

There are a lot of positioning methods for image target, the mature searching algorithm mainly used in face detection. In order to obtain the fire center coordinates, a regional connectivity marker detection using the left neighbor was proposed in the study. The method is to obtain each connected region, then operate regional calibration and merge, finally get the target fire connected region. The left-up neighborhood is shown in Fig .5. Referred to a pixel (i, j), on the left, left-up, up, and rightup, the 4 pixels $\{(\mathrm{i}, \mathrm{j}-1),(\mathrm{i}-1, \mathrm{j}-1),(\mathrm{i}-1, \mathrm{j})(\mathrm{i},-1, \mathrm{j}+1)\}$ constitute a set. It is only to judge the left-up pixel, unlike 4-neighborhood and 8-neighborhood which are also to determine the next line of adjacent pixels. Therefore, it scans the connected component labeling, and not repeats scanning, that would reduce the time consumption.

\begin{tabular}{|l|l|l|}
\hline$i-1, j-i 1-1, j$ & $i-1$ \\
\hline$i, j-1$ & $i, j$ & \\
\hline & & \\
\hline
\end{tabular}

Figure 5. Left-up neighbourhood.

After the binarizaton image of Fig .4 (d) was processed by the proposed algorithm, the edge contour of fire would be obtained. Then Canny is applied to the edge contour for obtaining the parameters of the fire. To find the centroid of fire in the edge image, the fire image coordinates were estimated taking the mean of all the coordinates of this labeled fire both $\mathrm{x}$ and y coordinates. Also, it implemented connected components labeling. Fig .6 gives edge contour of fire and finds localization of the fire center. Finally, the coordinates of the fire of each frame images were obtained and the open valve signal would output.

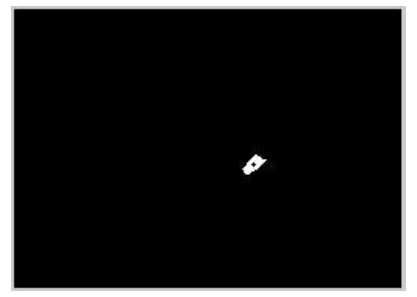

Figure 6. Fire center location.

\section{EXPERIMENT}

Because of the water gravity, jet placement and fire location point via video always exists deviation. On the basis of the fire location, according to the corresponding relation between the relative position for the fire source point in the image and the rotation direction of the proposed device, a compensating control method was used for device rotation, which is an angle adjustment from fine to heavy. That ensures the speed and precision positioning. The compensation parameters were gained through a series of experiments, which were shown in Table 1 . The relative angle is the upper and lower limit angle.

Experiments were conducted in a large test hall which is $30 \mathrm{~m}$ high, $42 \mathrm{~m}$ long and $20 \mathrm{~m}$ wide. The automatic jet fire extinguishing device was installed on one wall at the height of $12 \mathrm{~m}$. In the experiments, to test range, positioning and compensation angle for the device, the working pressure for the automatic jet fire extinguishing device was $0.8 \mathrm{Mpa}$, and the flow was $5 \mathrm{~L} / \mathrm{s}$. Using diesel as fuel, it is placed in a $0.2 \mathrm{~m} \times 0.2 \mathrm{~m} \times 0.05 \mathrm{~m}$ iron pool. The experimental setup is shown in Fig .7.

When the automatic jet fire extinguishing device receives the fire alarm signal, it will start to find the fire. In each test, the device started its search from the horizontal-left limit. The search process can be divided into three steps as searching fire source, positioning and compensating angle. Once the fire is into the camera view, the device will reduce the motor speed to process well fire image and gain fire center coordinates. After the positioning, compensation pitch angle will be chosen from Table 1, according to the initial parameters and vertical encoder pulse number of rotator motor. In the experiment, the automatic jet fire extinguishing device can locate and extinguish the test fire well.

TABLE I. THE COMPENSATION PARAMETERS (ANGLE UNIT: ${ }^{\circ}$ ).

\begin{tabular}{|c|c|c|c|c|c|c|}
\hline Angle & \multicolumn{5}{|c|}{ variable quantities between relative angle and compensation angle } \\
\hline Relative angle & $0 \sim 5$ & $5 \sim 10$ & $10 \sim 15$ & $15 \sim 20$ & $20 \sim 25$ & $25 \sim 30$ \\
\hline Compensation angle & 0 & 0 & 1 & 2 & 3 & 4 \\
\hline Relative angle & $30 \sim 35$ & $35 \sim 40$ & $40 \sim 45$ & $45 \sim 50$ & $50 \sim 55$ & $55 \sim 60$ \\
\hline Compensation angle & 6 & 8 & 10 & 12 & 15 & 18 \\
\hline Relative angle & $60 \sim 65$ & $65 \sim 70$ & $70 \sim 75$ & $75 \sim 80$ & $80 \sim 85$ & $85 \sim 90$ \\
\hline Compensation angle & 21 & 24 & 27 & 30 & 33 & 36 \\
\hline
\end{tabular}




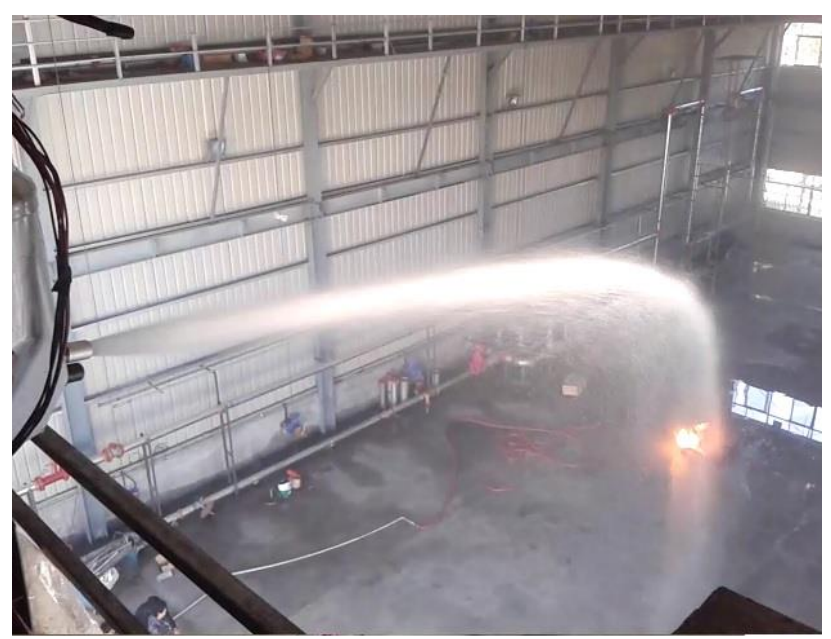

Figure 7. The test for automatic jet fire extinguishing device.

Moreover, the key influencing factors for the device is the jet pressure. Therefore, it is essential to revise the device initial parameters in different engineering.

\section{CONCLUSIONS}

Authors presented a video-based automatic jet fire extinguishing device. Then a fire detecting and locating method based on morphology and Left-up neighborhood algorithm was proposed and used for the device. To ensure the speed and precision positioning, a compensating control method was used for device rotation. Experiments were conducted in a large test hall to verify the videobased automatic jet fire extinguishing device. The results show that this method can locate and extinguish the test fire well.

The future work, accuracy of fire locating, reliability of moving parts, and compensating control method will be needed to improve.

\section{ACKNOWLEDGMENT}

This work was supported in part by the Fujian Science and Technology Key Project ( 2013H0038) , the Youth Foundation of Putian University (2013052), and the Educational Research Funds of Putian University (JG201309).

\section{REFERENCES}

[1] Guo TN, Fu ZM, "The fire situation and progress in fire safety science and technology in China," Fire Safety Journal, 2007, 42(3):171-182.

[2] Zhong MH, Fan WC, Liu TM, Zhang PH, Wei X, Liao GX, "China: some key technologies and the future developments of fire safety science," Safety Science, 2004, 42(7):627-637.

[3] Yu CY, Fang J,Wang JJ,Zhang YM, "Video fire smoke detection using motion and color features," Fire Technology, 2010, 46:651663.

[4] Chen XJ, Dong F, "A fire detecting method for video-based fire detector," Advanced Materials Research, 2014, 850-851:537-540.

[5] Vladimiras Suslavičius, Marijonas Bogdevičius, "New automatic impulse extinguishing device," Transport, 2008, 23(2):124-128.

[6] Liu JX, Chen XB, "Design of fire targeting device for large space auto tracking jet suppression system," Science and Technology Innovation Herald, 2013, 11:243-246.

[7] Chen T, Yuan HY, Su GF, Fan WC, "An automatic fire searching and suppression system for large spaces," Fire Safety Journal, 2004, 39:297-307.

[8] Xin YB, Thumuluru S, Jiang FH, Yin R, “An experimental study of automatic water cannon systems for fire protection of large open spaces," Fire Technology, 2014, 50:233-248.

[9] J. Serra, Image Analysis and Mathematical Morphology. New York: Academic Press, 1982.

[10] Chen T., Wu Q.H., Rahmani-Torkaman R., Hughes J., “A pseudo top-hat mathematical morphological approach to edge detection in dark regions," Pattern Recognition, 2002, 35(1):199-210. 